\title{
Bioactivities of berberine metabolites after transformation through CYP450 isoenzymes
}

\author{
Yi Li ${ }^{1 \dagger}$, Gang Ren ${ }^{1 \dagger}$, Yan-Xiang Wang ${ }^{1}$, Wei-Jia Kong ${ }^{1}$, Peng Yang ${ }^{1}$, Yue-Ming Wang ${ }^{1}$, Ying-Hong Li ${ }^{1}$, Hong Yi ${ }^{1}$, \\ Zhuo-Rong Li ${ }^{1}$, Dan-Qing Song ${ }^{1 *}$ and Jian-Dong Jiang ${ }^{1,2^{*}}$
}

\begin{abstract}
Background: Berberine (BBR) is a drug with multiple effects on cellular energy metabolism. The present study explored answers to the question of which CYP450 (Cytochrome P450) isoenzymes execute the phase-I transformation for BBR, and what are the bioactivities of its metabolites on energy pathways.

Methods: BBR metabolites were detected using LC-MS/MS. Computer-assistant docking technology as well as bioassays with recombinant CYP450s were employed to identify CYP450 isoenzymes responsible for BBR phase-I transformation. Bioactivities of BBR metabolites in liver cells were examined with real time RT-PCR and kinase phosphorylation assay.

Results: In rat experiments, 4 major metabolites of BBR, berberrubine (M1), thalifendine (M2), demethyleneberberine (M3) and jatrorrhizine (M4) were identified in rat's livers using LC-MS/MS (liquid chromatography-tandem mass spectrometry). In the cell-free transformation reactions, M2 and M3 were detectable after incubating BBR with rCYP450s or human liver microsomes; however, M1 and M4 were below detective level. CYP2D6 and CYP1A2 played a major role in transforming BBR into M2; CYP2D6, CYP1A2 and CYP3A4 were for M3 production. The hepatocyte culture showed that BBR was active in enhancing the expression of insulin receptor (InsR) and low-density-lipoprotein receptor (LDLR) mRNA, as well as in activating AMP-activated protein kinase (AMPK). BBR's metabolites, M1-M4, remained to be active in up-regulating InsR expression with a potency reduced by $50-70 \%$; LDLR mRNA was increased only by M1 or M2 (but not M3 and M4) with an activity level 35\% or $26 \%$ of that of BBR, respectively. Similarly, AMPK- $\alpha$ phosphorylation was enhanced by M1 and M2 only, with a degree less than that of BBR.
\end{abstract}

Conclusions: Four major BBR metabolites (M1-M4) were identified after phase-I transformation in rat liver. Cell-free reactions showed that CYP2D6, CYP1A2 and CYP3A4 seemed to be the dominant CYP450 isoenzymes transforming BBR into its metabolites M2 and M3. BBR's metabolites remained to be active on BBR's targets (InsR, LDLR, and AMPK) but with reduced potency.

\section{Background}

Berberine (BBR, Figure 1) is a natural compound isolated from Coptis chinensis and is for decades an overthe-count medicine in China for diarrhea [1]. Recently, accumulated research has identified BBR to be an effective drug in treating hyperlipidemia as well as hyperglycemia [2-4]. Clinical studies showed that oral administration of BBR caused significant reduction of

\footnotetext{
* Correspondence: songdanqingsdq@hotmail.com; jiang.jdong@163.com † Contributed equally

'Institute of Medicinal Biotechnology, Chinese Academy of Medical Sciences and Peking Union Medical College, Beijing 100050, China

Full list of author information is available at the end of the article
}

blood cholesterol, triglyceride as well as glucose in patients with hyperlipidemia and type 2 diabetes [2,3,5-7], with no side-effects on liver, kidney and muscle $[2,5]$. Mechanism studies have identified several important modes of action involved in the activities of BBR. The cholesterol-lowering effect was associated with extracellular-signal-regulated kinase (ERK) mediated LDLR mRNA up-regulation $[2,8]$; the glucoselowering effect mainly resulted from the protein kinase $\mathrm{C}$ (PKC) mediated InsR expression and the activation of AMPK $[3,4,9,10]$. The observed reduction of triglyceride by BBR might reflect its synergistic effect on both sugar and lipid metabolism [2-4].

\section{Biomed Central}


<smiles></smiles>

M1: berberrubine

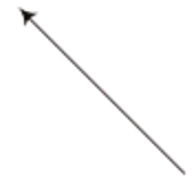

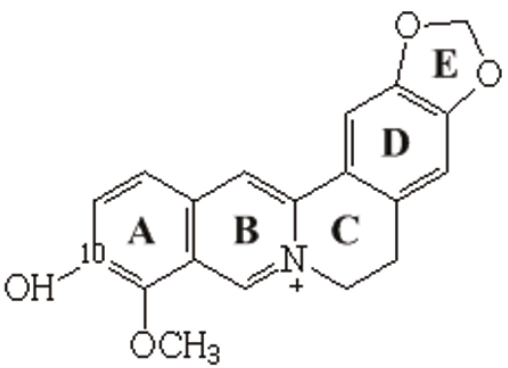

M2: thalifendine<smiles>COc1ccc2cc3n(c2c1)CCCc1cc(O)c(O)cc1-3</smiles>

M3: demethyleneberberine

BBR: berberine

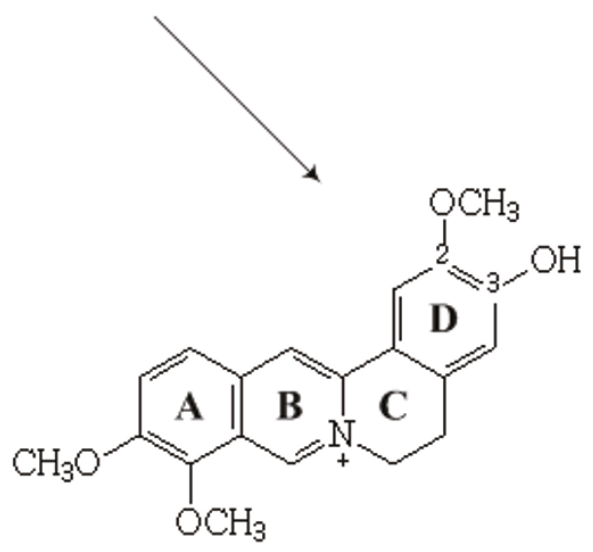

M4: jatrorrhizine

Figure 1 Chemical structure of berberine and its metabolites, berberrubine (M1), thalifendine (M2), demethyleneberberine (M3) and jatrorrhizine (M4).

At least 4 major metabolites of BBR have been identified in human blood after Phase I metabolism [11]; however, human CYP450 isoenzymes that are responsible for BBR phase-I transformation remain to be identified. Furthermore, bioactivities of the metabolites on the pathways mentioned above are also unknown. As most of our previous experiments for BBR were done in CYP450 positive human hepatocytes, HepG2 and Bel7402 [2-4], we were curious to learn which of the CYP450 isoenzymes are involved in BBR phase-I metabolism, and what are the bioactivities of the BBR metabolites. Answers to these questions might be important for patient selection in BBR clinical treatment as well as for chemical modification on BBR.

\section{Methods}

\section{Chemicals and reagents}

Berberine (BBR) chloride (purity, 98\%) was obtained from Sigma Chemical Co. (St. Louis, MO, USA). Berberrubine (M1), thalifendine (M2) and demethyleneberberine (M3) were synthesized by Chemical Department of our institute. All of the study compounds were in purity 
over 98.5\%. Jatrorrhizine (purity, 98\%) (M4) was purchased from the National Institute for the Control of Pharmaceutical and Biological Products (Beijing, China). The CYP450 inhibitors $\alpha$-naphthoflavone, quinidine and ketoconazole were from J\&K Chemical Ltd (Beijing, China). HPLC-grade acetonitrile and methanol were obtained from Fisher Scientific (New Jersey, USA). Pooled mixture of liver microsomes (Gentest Lot No. 70196, USA) and a NADPH-regenerating system were purchased from BD Gentest (Woburn, MA, USA). Microsomes from cDNA-transfected baculovirus-insect cells (expressing CYP1A2, CYP2A6, CYP2B6, CYP2C9, CYP2C19, CYP2D6, CYP2E1, CYP3A4 and CYP3A5) were also from the BD Gentest.

\section{Animal experiments}

Male Wistar rats (200-240 g, 8-week-old) purchased from Vital River Laboratories (Beijing, China) fasted overnight and were free access to water before drug administration. Rats were treated with with BBR orally (200 $\mathrm{mg} / \mathrm{kg}$ weight, $\mathrm{n}=8$ ) or distilled water as negative controls $(\mathrm{n}=3)$, and rat livers were removed $3 \mathrm{hrs}$ later. $2 \mathrm{~g}$ of the liver sample was washed with $5 \mathrm{~mL}$ normal saline, cut into small pieces and extracted with 3 -fold volume acetonitrile. After vortexed for $3 \mathrm{~min}$, the extract was centrifuged at $16000 \mathrm{~g}$ for $15 \mathrm{~min}$ at $4^{\circ} \mathrm{C}$ and the supernatant was diluted with $0.2 \%$ acetic acid. Ten microlitre dilution aliquot was injected into the LCMS/MS system for analysis.

\section{Structure-based BBR-CYP450 docking analysis}

The crystal structures of the six CYP450 isoforms were retrieved from the Protein Data Bank (PDB ID code 2HI4 for CYP1A2, solved at $1.95-\AA$ resolution [12]; $1 \mathrm{Z11}$ for CYP2A6, 2.05- $\AA$ [13]; 1OG5 for CYP2C9,

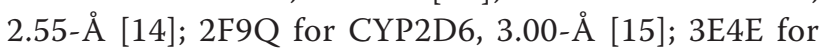
CYP2E1, 2.60- $\AA[16]$; 1 TQN for CYP3A4, 2.05- $\AA$ $[17,18])$. In this study, the docking was based on steric considerations but orienting the substrate recognition sites (SRSs) toward heme and ferryl oxygen of CYP450 protein $[19,20]$. A docking program FlexX (SYBYL 7.3, Tripos Inc) that uses an incremental construction algorithm was applied to optimize the interaction between the ligands and the substrate binding sites [21]. As for each of the CYP450 proteins, all crystal water molecules were removed from the original structure, hydrogen was added using Biopolymer module in SYBYL.

\section{BBR metabolism in human liver microsomes (HLMs)}

Incubation conditions were optimized to ensure the linearity with respect to the microsomal protein concentration and incubation time [22-24]. A typical incubation contained $0.1 \mathrm{mg}$ of human liver microsomes, $100 \mathrm{mM}$ phosphate buffer ( $\mathrm{pH}$ 7.4), BBR (dissolved in methanol, final volume of methanol $<0.1 \%$ [25]) and NADPHregenerating system (at final concentration of $3.3 \mathrm{mM}$ glucose-6-phosphate, $1.3 \mathrm{mM} \mathrm{NADP}{ }^{+}, 0.4$ unit $/ \mathrm{ml}$ glucose-6-phosphate dehydrogenase and $3.3 \mathrm{mM} \mathrm{MgCl} 2$ ) in a final volume of $200 \mu \mathrm{l}$. After pre-incubation of HLMs with $\mathrm{BBR}$ for $5 \mathrm{~min}$ at $37^{\circ} \mathrm{C}$, the reaction was initiated by addition of NADPH-regenerating system. After incubation of the mixture in a $37^{\circ} \mathrm{C}$ water bath with opening to the air, the reaction lasted for $30 \mathrm{~min}$ and was terminated by addition of $800 \mu \mathrm{l}$ cold acetonitrile. Internal standard ( $40 \mu \mathrm{g} / \mathrm{ml}$ ampicillin, dissolved in acetonitrile) was added into the mixture, and then centrifuged at $16000 \mathrm{~g}$ for $15 \mathrm{~min}$ at $4^{\circ} \mathrm{C}$. The supernatant was aspirated, followed by dilution in $0.2 \%$ acetic acid. $10 \mu \mathrm{l}$ of the aliquot was injected into the LC-MS/MS system for analysis. Control samples were incubated without $\mathrm{NADPH}$. The incubation was performed in duplicates.

\section{BBR metabolism in recombinant human CYP450 isoenzymes}

The experiment was performed with the condition described in the method using HLMs for transformation. $50 \mathrm{pmol} / \mathrm{mL}$ of each of the CYP450 isoenzymes incubated with $20 \mu \mathrm{mol} / \mathrm{L}$ BBR. Control microsome prepared from insect cells, which were infected with wildtype baculovirus, severed as a negative control. The incubation was performed in duplicates.

\section{Chemical inhibition assays}

$\alpha$-Naphthoflavone [26] (typical inhibitor of CYP1A2; final concentration used, $5 \mu \mathrm{M}$ ), quinidine $[27,28]$ (typical inhibitor of CYP2D6; final concentration, $5 \mu \mathrm{M}$ ) sulfaphenazole [23] (typical inhibitor of CYP2C9; final concentration, $5 \mu \mathrm{M}$ ), troglitazone [23] (typical inhibitor of CYP2C19; final concentration, $5 \mu \mathrm{M}$ ) and ketoconazole [29] (typical inhibitor of CYP3A; final concentration, $5 \mu \mathrm{M}$ ) were added, respectively, to the incubation reaction containing $0.5 \mathrm{mg} / \mathrm{ml}$ HLMs, $100 \mathrm{mM}$ phosphate buffer ( $\mathrm{pH}$ 7.4) and NADPH-regenerating system at a final volume of $200 \mu \mathrm{l}$. Before addition of BBR (15 $\mu \mathrm{M})$, the samples were pre-incubated for $10 \mathrm{~min}$ at $37^{\circ} \mathrm{C}$ and transferred onto ice for $45 \mathrm{~min}$. The mixture was incubated at $37^{\circ} \mathrm{C}$ for $30 \mathrm{~min}$ and then terminated with cold acetonitrile [30]. Sample analysis was described above. The incubations were performed in duplicates.

\section{Kinetic experiments}

To measure the enzyme kinetic parameters both in HLMs and rCYP450s, we adjusted the standard incubation mixture containing BBR at a final concentration between $0.15 \sim 75 \mu \mathrm{M}$. Incubation conditions were as described above. Samples were analyzed by LC-MS/MS. The kinetic parameters Vmax and $\mathrm{Km}$ were calculated using EK1 v10.0 program (SPSS Inc., Chicago, IL, USA), 
and these values were used to calculate the intrinsic clearance (Clint, $V \max / \mathrm{Km}$ ). The results were expressed as mean $\pm \mathrm{sd}$ from three independent experiemnts. The percentage of total normalized rate (\%TNR) was calculated to estimate the contribution of each CYP450 in BBR transformation [22].

\section{LC-MS/MS analysis}

Sample analysis was performed using an LC-MS/MS system (Thermo-Finnigan, San Jose, CA, USA), which consisted of a Surveyor LC pump with an on-line degasser, a Surveyor autosampler and a TSQ Quantum triplequadrupole mass spectrometer equipped with an ESI probe. A Waters XTerra ${ }^{\circledR}$ MS $C_{18}(2.1 \times 50 \mathrm{~mm}, 3.5$ $\mu \mathrm{m})$ column (Milford, MA, USA) was used for separation at $25^{\circ} \mathrm{C}$. The mobile phase consisted of $0.2 \%$ acetic acid in water (A) and 100\% acetonitrile (B). The flowrate was $0.2 \mathrm{ml} / \mathrm{min}$. In gradient elution, the proportion of acetonitrile (B) was linearly increased from $10 \%$ to $100 \%$ in $18 \mathrm{~min}$, held at $100 \%$ for $2 \mathrm{~min}$ and returned to $10 \%$ in $0.1 \mathrm{~min}$. The column was allowed to equilibrate for $10 \mathrm{~min}$ after each run. Only the data from $2 \mathrm{~min}$ to 15 min was acquired by MS. The MS was operated in positive ESI mode. Nitrogen was used as both the sheath and auxiliary gas at a pressure of 35 and 10 arbitrary units, respectively. The spray voltage was set at $4.0 \mathrm{kV}$ and the capillary temperature was at $270^{\circ} \mathrm{C}$. Full scan and product ion mass spectra results of BBR as well as M1-M4 were obtained (data are not shown). The most abundant product ion of each chemical was chosen for selected reaction monitoring (SRM). The SRM transitions and collision energies are shown in Table 1. Data were analyzed by Xcalibur 1.2 software.

\section{Cell culture and drug treatment}

The Human hepatoma cell line HepG2 was obtained from the American Tissue Culture Collection (ATCC, USA). HepG2 cells were grown in Eagle's Minimum Essential Medium (GIBCO) supplemented with 10\% fetal bovine serum (GIBCO), $1 \%$ non-essential amino acids (GIBCO) and $1 \%$ antibiotics (100 units/ml of penicillin and $100 \mu \mathrm{g} / \mathrm{ml}$ of streptomycin), and incubated at $37^{\circ} \mathrm{C}$ in a humidified atmosphere with $5 \% \mathrm{CO}_{2}$.

Table 1 SRM transitions and collision energies used in LC-MS/MS for the detection of BBR, M1, M2, M3 and M4

\begin{tabular}{cccc}
\hline Chemical & $\begin{array}{c}\text { Molecular mass } \\
(\mathbf{M W})\end{array}$ & $\begin{array}{c}\text { SRM transition } \\
(\mathbf{m} / \mathbf{z})\end{array}$ & $\begin{array}{c}\text { Collision energy } \\
(\mathrm{eV})\end{array}$ \\
\hline BBR & 335 & $336.0 \rightarrow 319.8$ & 28 \\
M1 & 321 & $322.0 \rightarrow 306.8$ & 31 \\
M2 & 321 & $322.0 \rightarrow 306.8$ & 31 \\
M3 & 323 & $324.0 \rightarrow 307.8$ & 32 \\
M4 & 338 & $338.0 \rightarrow 321.9$ & 30 \\
\hline
\end{tabular}

For real time RT-PCR and double immune-staining analysis, HepG2 cells were starved in Eagle's MEM supplemented with $0.5 \%$ FBS overnight and treated with BBR or its metabolites (M1-M4) for 8 hrs, respectively. For immunoblot assay, cells were starved in serum-free medium overnight and treated with BBR or its metabolites (M1-M4) for $24 \mathrm{hrs}$. The study compounds were diluted in culture medium prior to use.

\section{RNA isolation and real-time RT-PCR}

Total cellular RNAs were isolated by the Ultraspec RNA lysis solution (Biotecxs Laboratory, Houston, TX) by following the vender's instruction. Total RNAs were reversely transcribed into cDNAs using the Reverse Transcription System (Promega, Madison, WI). Quantitative real time PCR was performed with these cDNAs as described previously [2], with $\beta$-Actin as an internal control. Normalized InsR or LDLR mRNA expression levels were plotted as fold of the untreated control. The primers used were described previously $[2,3]$.

\section{Double immune-staining and flow cytometry analysis}

HepG2 cells were treated with BBR $(27 \mu \mathrm{M})$, simvastatin $(1 \mu \mathrm{M})$ or rosiglitazone $(10 \mu \mathrm{M})$ for 8 hrs, respectively. The cells were harvested, washed in PBS and incubated for $1 \mathrm{hr}$ at $37^{\circ} \mathrm{C}$ in the presence of both monoclonal antibody to InsR (Labvision/NeoMarkers, Fremont, CA) and rabbit polyclonal antibody against LDLR (Santa Cruz Biotechnology, Santa Cruz, CA). The corresponding isotype-matched, nonspecific mouse and rabbit IgGs were used respectively as controls for nonspecific staining. After washing in PBS, cells were stained with a fluorescein isothiocyanate (FITC)-conjugated goat antimouse IgG (green color, Santa Cruz Biotechnology), as well as a tetramethyl rhodamine isothiocyanate (TRITC)-conjugated goat anti-rabbit IgG (red color, Santa Cruz Biotechnology). The fluorescence intensities were analyzed in a FACS (FACSort, Becton Dickinson).

\section{Immunoblot}

Sample cells were rinsed with phosphate-buffered saline and lysed in SDS-PAGE loading buffer. Cell lysates were subjected to $8 \%$ SDS-PAGE for protein separation, and protein bands were transferred onto polyvinylidene difluoride membranes (Millipore). The amount of protein and extent of phosphorylation were estimated using the following primary rabbit antibodies, anti-phosphoAMPK- $\alpha$-Thr-172 antibody, anti-AMPK- $\alpha$ antibody as well as anti- $\beta$-actin antibody (Cell signaling Technology, USA). The secondary antibody was peroxidase-conjugated goat anti-rabbit antibody (Cell signaling Technology, USA). After binding, the bands were revealed with enhanced chemiluminescence using the ECL commercial kit (Millipore). 


\section{Statistics}

Differences of mean results among study groups were examined by a two-tailed unpaired Student's $t$-test for equal or unequal variances depending on a preliminary F test for homogeneity of variance. Results are expressed as the means \pm sd. $\mathrm{P}<0.05$ was considered significant.

\section{Results and Discussion}

\section{BBR metabolites are detectable in rat's liver tissue}

It has been documented that BBR has four major metabolites after phase I metabolism in vivo, berberrubine (M1), thalifendine (M2), demethyleneberberine (M3) and jatrorrhizine (M4) [31]. The chemical structures of the four compounds are shown in Figure 1. These metabolites were detectable in the blood and urine in human and rodents $[11,31]$. The biotransformation of BBR in rats was reported to be similar to that in human [31]. As BBR's activity was examined in hepatocytes in our previous experiments [2-4], we detected the BBR metabolites in rat liver to address the question of whether hepatocytes contain these metabolized products. In the present study, the 4 metabolites M1, M2, M3 and M4 were all detectable in rat livers $3 \mathrm{hrs}$ after BBR oral administration (Figure 2). The highest level was seen in M2 and the lowest in M1. The result was consistent with the previous reports of BBR metabolites in rodent blood [11,31].

\section{CYP450 isoenzymes that involved in BBR phase I metabolism}

For BBR's metabolite formation in liver, our question was which of the CYP450 isoenzymes transform BBR into its metabolites. Thus, nine major recombinant human CYP450 isoenzymes (CYP1A2, CYP2A6, CYP2B6, CYP2C9, CYP2C19, CYP2D6, CYP2E1, CYP3A4 and CYP3A5) were investigated in this study. Of the nine isoenzymes, six (CYP1A2, CYP2A6, CYP2C9, CYP2D6, CYP2E1 and CYP3A4) have their 3D structural database available from PDB (Protein Data Bank). As the initial step, computer-assistant docking analysis was performed using SYBYL 7.3 system for the affinity between BBR and each isoenzyme. As shown in Figure 3a for the docking score, the CYP2D6, CYP1A2 and CYP3A4 exhibited considerably good docking performance with respect to the positive docking cut-off value set at 5 . The molecular interactions between BBR and the three CYP450s are shown in Figure 3b. BBR anchored in the binding site of CYP2D6 through hydrogen bond interaction with the side chain of Arg-221; Phe-120 played a major role in controlling the orientation of BBR to the heme; the Phe-483 made a cross lock to the $\mathrm{C}$ - and D-ring of BBR (Figure 3b, upper). The substrate binding cavity of CYP1A2 was uniformly narrow throughout its extent and was lined by residues Gly-316-Ala-317 and the Asp-320 peptide bond, which constituted a relatively planar substrate binding platform. Phe-226 produced another parallel substrate binding surface and a $\pi-\pi$ stacking with BBR. In addition, both orthogonal and parallel aromatic interactions between BBR and residues Phe-125 and Phe-226 contributed to a tight binding affinity. Also, a water molecule of CYP1A2 hydrogen-bonding to BBR and Gly-316 strengthens this binding (Figure $3 \mathrm{~b}$, middle). In the BBR-CYP3A4 docking pattern, hydrogen bonding and orthogonal aromatic interactions were observed between

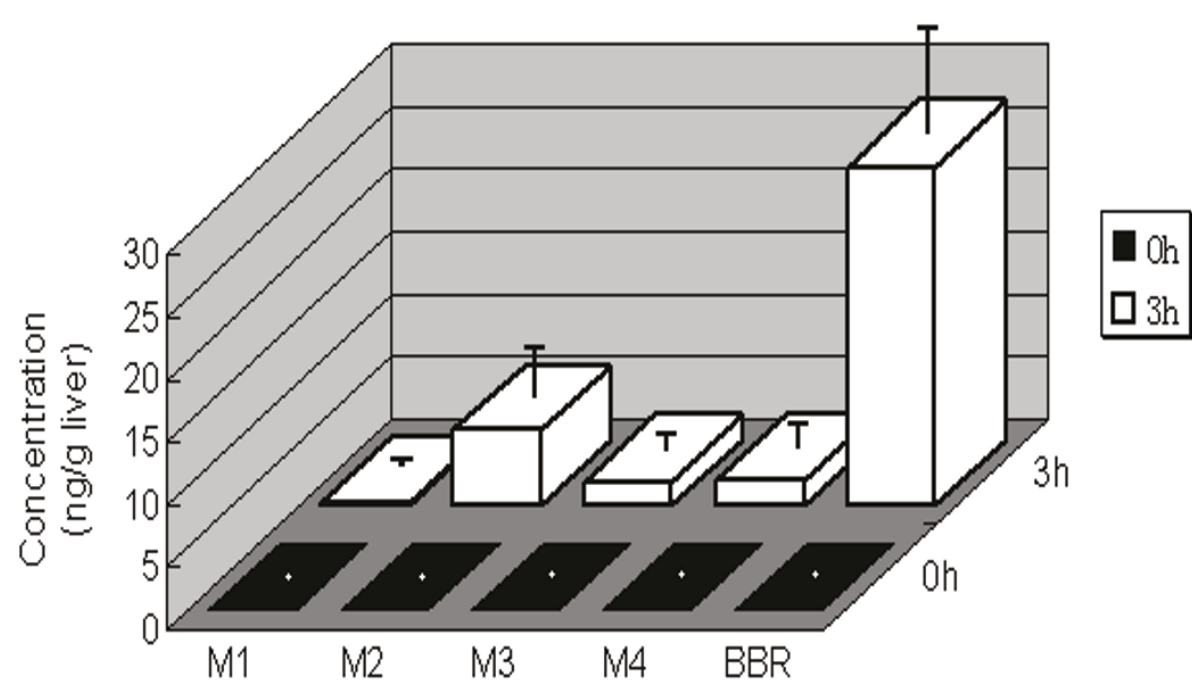

Figure 2 BBR and its main phase-I metabolites in rat liver. Male Wistar rats (200-240 g, 8-week-old) were orally treated with BBR (200 mg/ $\mathrm{kg}$ ). Rat livers $(n=8)$ were removed 3 hrs later, followed by homogenization, cell lysate extraction and LC MS/MS detection. Presented are mean and sd of the 8 rats. Untreated control rats $(n=8)$ were used for comparison. 

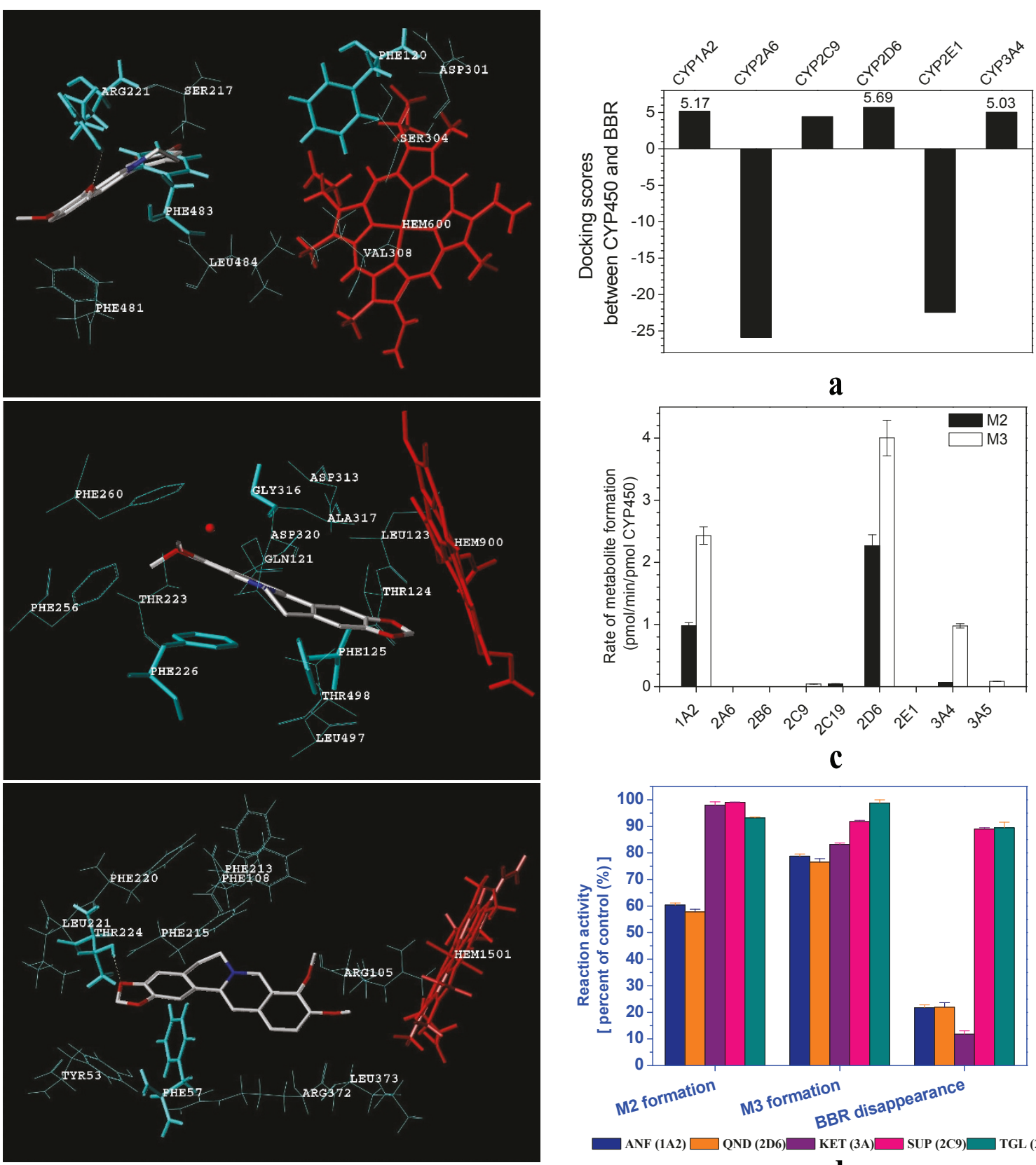

b

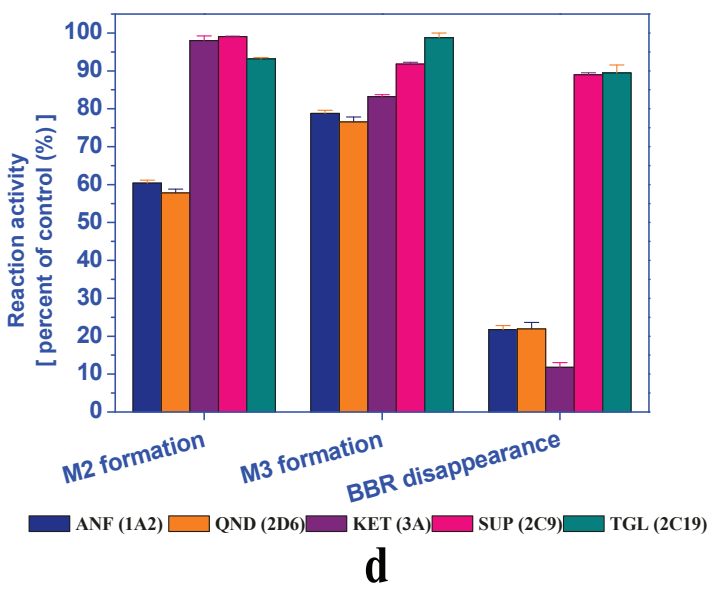

Figure 3 Identification of CYP450 isoenzymes that transform BBR into its metabolites. Interaction between CYP450 and BBR were analyzed with docking score generated from SYBYL 7.3 software analysis. CYP450 isoenzymes with docking scores over 5 were labelled with the score value (a). The 3-D structrual docking patterns between BBR and CYP2D6 (upper), or CYP1A2 (middle), or CYP3A4 (lower) were generated with SYBYL 7.3. BBR is rendered in sticks (red, oxygen atoms; white, carbon atoms; blue, nitrogen atoms). The heme prosthetic group is rendered in sticks in red. The amino acid residues constituting the active site cavity are in cyan, most of which are shown in lines. Amino acid residues rendered in sticks may help BBR binding to CYP450. Specific hydrogen bonds and a water molecule are showed as yellow broken lines and a red sphere, respectively (b). For the transformation test in rCYP450 isoenzymes reaction system, BBR $(20 \mu \mathrm{M})$ was incubated with each of the rCYP450 isoenzymes ( $50 \mathrm{pmol} / \mathrm{ml}$ of rCYP450) for $0.5 \mathrm{hr}$, followed by a detection of the metabolites. The experiment was repeated twice (c). In the chemical inhibition assay, the $\alpha$-naphthoflavone (ANF) was for CYP1A2 inhibition, quinidine (QND) for CYP2D6, ketoconazole (KET) for CYP3A, sulfaphenazole (SUP) for CYP2C9 and troglitazone (TGL) for CYP2C19. BBR was incubated with HLMs in the presence or absence of CYP450 specific inhibitors. The final concentration of BBR and the inhibitor in the reaction was $15 \mu \mathrm{M}$ and $5 \mu \mathrm{M}$, respectively. The enzyme catalyzing activity of sample free of inhibitors (control) was defined as 100\%. Presented is mean and sd of the percent of control (d). 
BBR and the residues Thr-224 and Phe-57, respectively (Figure 3b, lower). The results suggest that the three cellular CYP450 isoenzymes might have good binding affinity to BBR.

Then, the docking experiment was corroborated with biochemical assay. Each of the above-mentioned CYP450 isoenzymes was in turn incubated with BBR for $30 \mathrm{~min}$, followed by detection of BBR metabolites with LC-MS/MS. The results are shown in Figure 3c. Treatment of BBR with CYP2D6 and CYP1A2 generated a considerable level of M2 and M3, suggesting that they were the dominant contributors to transform BBR into M2 and M3. CYP3A4 was also important for the production of M3 but only with a minor role in M2 production. In addition, CYP2C19, CYP2C9 and CYP3A5 showed some very weak effect in producing M2 and M3, respectively. CYP2A6, CYP2B6 and CYP2E1 were without effect on BBR transformation. Although the experiment was repeated for over 5 times, M1 and M4 were not detectable in the reaction system.

If the results were true, inhibition of the CYP450 isoenzymes should reduce the formation of the metabolites. Therefore, chemical inhibition assays were carried out in the HLMs reaction system for the CYP2D6-, CYP1A2-, CYP3A-, CYP2C9-, or CYP2C19-mediated BBR transformation, respectively. BBR was treated with HLMs for $30 \mathrm{~min}$ in the presence or absence of specific CYP450 isoenzyme inhibitors. The effects of the inhibitors on BBR metabolism in HLMs reaction were shown in Figure $3 \mathrm{~d}$. The reaction activity of the control sample (with no inhibitor) was defined as $100 \%$ for the comparison with that of the samples treated with inhibitors. M2 formation was decreased by about $40 \%$ after ANF (for CYP1A2) or QND (for CYP2D6) treatment, but not KET treatment (for CYP3A); M3 formation decreased by around $20 \%$ after treatment with inhibitor for CYP1A2, or CYP2D6, or CYP3A; inhibitors for CYP2C9 and CYP2C19 showed only minor or no effect. Accordingly, BBR disappearance was found decreased in the reactions treated with inhibitors for CYP1A2, CYP2D6 and CYP3A, but not with those for CYP2C9 and CYP2C19. The sum of reduction in the formation of M2 and M3 were close to the reduction of berberine disappearance (Figure $3 \mathrm{~d}$ ). The results showed the role of the three CYP450 isoenzymes in the BBR phase-I metabolism, and were consistent with that from computer docking. The inhibition rate of each of the inhibitors for CYP1A2, CYP2D6 and CYP3A was in the range between $15-40 \%$. The inhibitory efficacy of each of the inhibitors was not strong (especially for the inhibition of M3 formation), indicating a combined effect of the three major isoenzymes in BBR's transformation.

Considering the relative expression of these CYP450 isoforms in liver, enzyme kinetic study using these
CYP450 isoenzymes was performed and the relative contribution of each of the CYP450 isoenzymes was calculated. The results were in Table 2 and 3. For M2 production, the contribution rate of CYP1A2, CYP2D6 and CYP3A4 was $72.07 \%, 25.21 \%$ and $2.72 \%$, respectively. For M3, the contribution rate was $26.36 \%, 37 \%$ and $36.63 \%$ for CYP1A2, CYP2D6 and CYP3A4, respectively.

\section{Activity of the BBR metabolites on InsR, LDLR and AMPK}

Next, each of the BBR metabolite compounds was examined for their effect on the expression of InsR and LDLR mRNA, as well as on the activity of AMPK in human hepatocytes. For LDLR mRNA expression, the original $\mathrm{BBR}$ remained to be the strongest up-regulator (Figure 4a); among the 4 metabolites, M1 and M2 remained to be active with an activity $35 \%$ and $26 \%$ of that of $B B R$, respectively; $M 3$ and M4 were without effect. Although the 4 metabolites were active in increasing InsR mRNA level in HepG2 hepatocytes by around 1.5 folds, the original form of BBR appeared to be the most active one up-regulating InsR mRNA level by over 2 folds (Figure 4b). It suggests that BBR in its original form is the most potent functional compound for the hypoglycemic as well as lipid-lowering effect in clinic. To compare BBR's effect with the known type 2 diabetes drug rosiglitazone and anti-cholesterol drug simvastatin, double immune-staining was conducted for the cell-surface protein expression of InsR and LDLR. The results demonstrated an up-regulatory effect of BBR on both LDLR and InsR on the membrane of human hepatocytes, suggesting a unique effect of BBR on the two major molecules related to energy metabolism. In contrast, simvastatin increased LDLR expression only, but not InsR; and rosiglitazone showed effect on neither LDLR nor InsR (Figure 4c). The results hint an advantage of BBR over the known drugs in modulating cellular energy metabolism.

AMPK's activity closely associates with sugar metabolism. Kim et al has reported that BBR reduced blood glucose through activation of AMPK in adipose cells [9]. Here, we tested BBR metabolites for their activity on this kinase in liver cells. As shown in Figure 4d, among

Table 2 CYP450s responsible for M2 formation

\begin{tabular}{llll}
\hline & CYP1A2 & CYP2D6 & CYP3A4 \\
\hline Km $(\mu \mathrm{M})$ & $100.0 \pm$ & $31.9 \pm$ & $27.8 \pm 0.79$ \\
& 8.74 & 1.23 & \\
Vmax (pmol/min/pmol P450) & $5.4 \pm$ & $2.7 \pm$ & $0.024 \pm$ \\
& 0.094 & 0.0052 & 0.0021 \\
Clint ( $\mathrm{LL} / \mathrm{min} / \mathrm{pmol}$ P450) & 0.054 & 0.085 & 0.00085 \\
Nominal P450 Content in HLMs & 45 & 10 & 108 \\
(pmol/mg) & & & \\
Adjusted Clint ( $\mu \mathrm{L} / \mathrm{min} / \mathrm{mg})$ & 2.43 & 0.85 & 0.0918 \\
Percentage Adjusted Clint $(\%)$ & 72.07 & 25.21 & 2.72 \\
\hline
\end{tabular}


Table 3 CYP450s responsible for M3 formation

\begin{tabular}{llll}
\hline & CYP1A2 & CYP2D6 & CYP3A4 \\
\hline $\mathrm{Km}(\mu \mathrm{M})$ & $125.8 \pm$ & $12.0 \pm$ & $27.1 \pm$ \\
& 3.57 & 0.68 & 1.03 \\
Vmax (pmol/min/pmol P450) & $4.8 \pm 0.54$ & $2.9 \pm$ & $0.59 \pm$ \\
& & 0.19 & 0.012 \\
Clint ( $\mu \mathrm{L} / \mathrm{min} / \mathrm{pmol}$ P450) & 0.038 & 0.24 & 0.022 \\
$\begin{array}{l}\text { Nominal P450 Content in HLMs } \\
\text { (pmol/mg) }\end{array}$ & 45 & 10 & 108 \\
Adjusted Clint ( $\mu$ L/min/mg) & 1.71 & 2.4 & 2.376 \\
Percentage Adjusted Clint (\%) & 26.36 & 37.0 & 36.63 \\
\hline
\end{tabular}

the study compounds BBR was again the most active compound for AMPK activation; the treatment of HepG2 human liver cells with M1 and M2 increased the activity of AMPK but with a less potency as compared to that of BBR; the effect of M3 and M4 on this kinase appeared to be not significant. The experiment was repeated for more than 3 times and the results were reproducible. As the BBR bioactivities were kept at certain degrees in the metabolites with an intact $\mathrm{E}$ ring (M1 and M2), but abolished in those with $\mathrm{E}$ ring opened
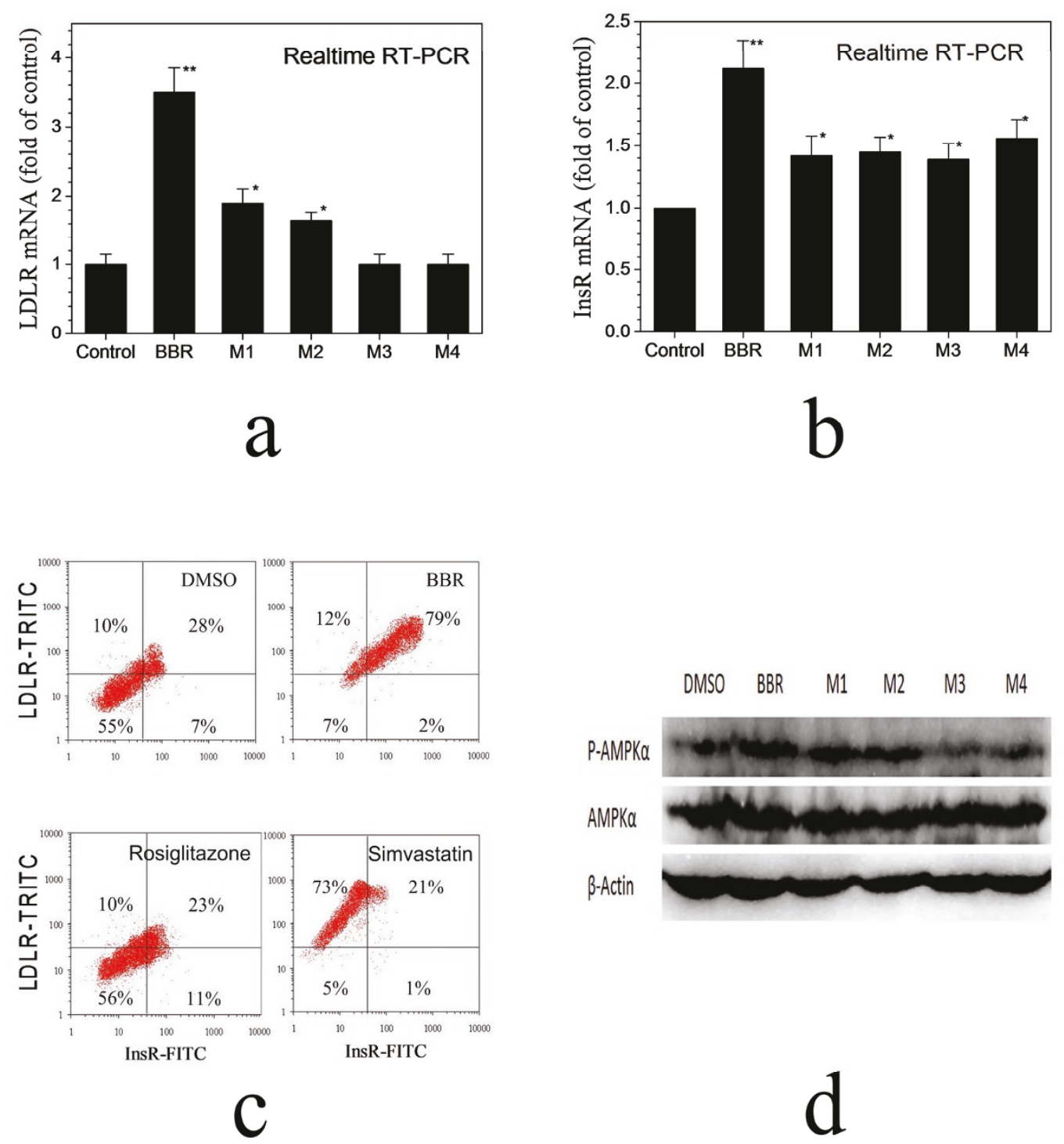

Figure 4 Effect of BBR metabolites on InsR, LDLR and AMPK. HepG2 cells were respectively treated with the compounds for 8 hrs at concentration of $20 \mu \mathrm{M}$ for InsR test or $40 \mu \mathrm{M}$ for LDLR, followed by RNA extraction and real time RT-PCR assay for the InsR or LDLR mRNA. The amount of LDLR (a) and InsR (b) mRNA in the treated cells was normalized to that of the untreated control. ${ }^{*} p<0.05$, ${ }^{* *} p<0.01$, vs the untreated control. To detect the protein expression, HepG2 cells were treated with BBR $(27 \mu \mathrm{M})$ for 8 hrs, with simvastatin $(1 \mu \mathrm{M})$ and rosiglitazone $(10 \mu \mathrm{M})$ as references. Berberine increased the cell-surface expression of both InsR and LDLR in the hepatocytes (c). For AMPK activation, HepG2 cells were treated with the study compounds $(20 \mu \mathrm{M})$ for 24 hrs with DMSO as a control. AMPK-alpha phosphorylation in the HepG2 cells was detected with immunoblots, using a protocol described in the Methods (d). 
(M3 and M4), the presence of E ring on BBR (Figure 1) seems to be essential for their activity on LDLR and AMPK, consistent with our previous observation [32]. The analysis of the functional group of BBR has led us to a creation of an M1 pro-drug, which demonstrated an increased efficacy as compared with BBR [33].

$B B R$ is a safe medicine with multiple therapeutic effects. This compound has a poor absorption rate in human gut with less than $10 \%$ of BBR entering into blood stream [34]. BBR were metabolized into at least four metabolites after phase-I metabolism in vivo. This study shows for the first time that CYP1A2, CYP2D6 and CYP3A4 are the major contributors for the transformation of BBR into its metabolites in human liver microsomes. The main metabolites detectable in the enzymatic reaction were $\mathrm{M} 2$ and $\mathrm{M} 3 ; \mathrm{M} 1$ and $\mathrm{M} 4$ were not found after incubation of BBR with recombinant CYP450s or the HLMs mixture. As the sensitivity of our LC-MS/MS test was in 100 500 pg range, the M1 and M4 content might be under this level in the reactions. Further optimization of the experimental condition might help us to identify these two metabolites although their concentration seemed to be extremely low in these reactions. It is worthy to note that statins are mainly metabolized under the action of CYP3A4 [35,36], relatively different from that in BBR. The information might be helpful in designing clinical therapeutic regimen combining statins with BBR [7].

Also, this study shows for the first time that although BBR itself is the most active compound to up-regulate InsR expression, all of its metabolites remain to be active at certain degree on this target. For LDLR up-regulation and AMPK activation, M1 and M2 are the two active metabolites, with reduced potency. The study suggests that the action of BBR on cellular energy metabolism pathways mainly originates from BBR in its original form.

\section{Conclusions}

Taken together, four major BBR metabolites (M1-M4) were identified after phase-I transformation in rat liver. CYP1A2, CYP2D6 and CYP3A4 appeared to be the key enzymes to transform BBR into M2 and M3 in cell-free metabolism reactions. The metabolites of BBR remained to be active on InsR (by M1-4), LDLR (by M1 and M2), and AMPK (by M1 and M2), but with largely reduced potency. It appears that BBR in its original form is the active chemical entity that regulates multiple pathways in energy metabolism.

\section{List of abbreviations}

BBR: berberine; M1: berberrubine; M2: thalifendine; M3: demethyleneberberine: M4: jatrorrhizine; HLMs: human liver microsomes; rCYP450: recombinant human cytochrome P450; LC-MS/MS: liquid chromatography - tandem mass spectrometry; NADPH: reduced nicotinamide adenine dinucleotide phosphate; InsR: insulin receptor; LDLR: low-density-lipoprotein-receptor; AMPK: AMP-activated protein kinase; ERK: extracellular-signal-regulated kinase; PKC: protein kinase C.

\section{Acknowledgements}

This work was supported by the National Natural Science Foundation of China (90913002) and the "National S\&T Major Special Project on Major New Drug Innovation" (2009ZX09301-003).

\section{Author details}

${ }^{1}$ Institute of Medicinal Biotechnology, Chinese Academy of Medical Sciences and Peking Union Medical College, Beijing 100050, China. ${ }^{2}$ State Key Laboratory of Bioactive Substances and Functions of Natural Medicines, Institute of Materia Medica, Chinese Academy of Medical Sciences and Peking Union Medical College, Beijing 100050, China.

\section{Authors' contributions}

LY carried out the CYP450 metabolism study, computer-aided docking study, rat experiment and participated in writing of the manuscript; RG carried out the AMPK activity assay and participated in writing of the manuscript; KWJ carried out the LDLR and InSR expression analysis; YP, WYX and LYH did the synthesis and analysis of M1, M2 and M3; YH and LZR participated in the computer-aided design assay; JJD and SDQ designed and organized the studies, analysed data and wrote the manuscript. All of the authors read and approved the final manuscript.

\section{Competing interests}

The authors declare that they have no competing interests.

Received: 1 January 2011 Accepted: 15 May 2011

Published: 15 May 2011

\section{References}

1. Zhao GP: Huanglian. In Chinese Materia Medica Dictionary. Volume 2.. 2 edition. Edited by: Zhao GP. Shanghai: Science and Technology Press; 2006:2815-2820.

2. Kong WJ, Wei J, Abidi $P$, Lin M, Inaba S, Li C, Wang Y, Wang Z, Si S, Pan H, Wang S, Wu J, Wang Y, Li Z, Liu J, Jiang JD: Berberine is a Novel Cholesterol-Lowering Drug Working Through a Unique Mechanism Distinct from Stains. Nat Med 2004, 10:1344-1351.

3. Zhang H, Wei J, Xue R, Wu JD, Zhao W, Wang ZZ, Wang SK, Zhou ZX, Song DQ, Wang YM, Pan HN, Kong WJ, Jiang JD: Berberine lowers blood glucose in type 2 diabetes mellitus patients through increasing insulin receptor expression. Metabolism 2010, 59:285-292.

4. Kong WJ, Zhang H, Song DQ, Xue R, Zhao W, Wei J, Wang YM, Shan N, Zhou ZX, Yang P, You XF, Li ZR, Si SY, Zhao LX, Pan HN, Jiang JD: Berberine reduces insulin resistance through protein kinase Cdependent up-regulation of insulin receptor expression. Metabolism 2009, 58:109-119.

5. Zhao W, Xue R, Zhou ZX, Kong WJ, Jiang JD: Reduction of blood lipid by berberine in hyperlipidemic patients with chronic hepatitis or liver cirrhosis. Biomed Pharmacother 2008, 62:730-731.

6. Zhang Y, Li X, Zou D, Liu W, Yang J, Zhu N, Huo L, Wang M, Hong J, Wu P, Ren G, Ning G: Treatment of Type 2 Diabetes and Dyslipidemia with the Natural Plant Alkaloid Berberine. J Clin Endocrinol Metab 2008, 93:2559-2265.

7. Kong WJ, Wei J, Zuo ZY, Wang YM, Song DQ, You XF, Zhao LX, Pan HN, Jiang JD: Combination of simvastatin with berberine improves the lipidlowering efficacy. Metabolism 2008, 57:1029-1037.

8. Abidi P, Zhou Y, Jiang JD, Liu J: Extracellular signal-regulated kinasedependent stabilization of hepatic low-density lipoprotein receptor mRNA by herbal medicine berberine. Arterioscler Thromb Vasc Biol 2005 , 25:2170-2176.

9. Lee $Y S$, Kim WS, Kim KH, Yoon MJ, Cho HJ, Shen Y, Ye JM, Lee $\mathrm{CH}$, Oh WK, Kim CT, Hohnen-Behrens C, Gosby A, Kraegen EW, James DE, Kim JB: Berberine, a natural plant product, activates AMP-activated protein kinase with beneficial metabolic effects in diabetic and insulin-resistant states. Diabetes 2006, 55:2256-2264

10. Yin J, Gao Z, Liu D, Liu Z, Ye J: Berberine improves glucose metabolism through induction of glycolysis. Am J Physiol Endocrinol Metab 2008, 294 E148-E156. 
11. Zuo F, Nakamura N, Akao T, Hattori M: Pharmacokinetics of berberine and its main metabolites in conventional and pseudo germ-free rats determined by liquid chromatography/ion trap mass spectrometry. Drug Metab Dispos 2006, 34:2064-2072

12. Sansen S, Yano JK, Reynald RL, Schoch GA, Griffin KJ, Stout CD, Johnson EF: Adaptations for the Oxidation of Polycyclic Aromatic Hydrocarbons Exhibited by the Structure of Human P450 1A2. J Biol Chem 2007, 282:14348-1455.

13. Sansen S, Hsu MH, Stout CD, Johnson EF: Structural insight into the altered substrate specificity of human cytochrome P450 2 A6 mutants. Arch Biochem Biophys 2007, 464:197-206.

14. Wester MR, Yano JK, Schoch GA, Yang C, Griffin KJ, Stout CD, Johnson EF: The Structure of Human Cytochrome P450 2C9 Complexed with Flurbiprofen at 2.0-A Resolution. J Biol Chem 2004, 279:35630-35637.

15. Rowland P, Blaney FE, Smyth MG, Jones JJ, Leydon VR, Oxbrow AK, Lewis CJ, Tennant MG, Modi S, Eggleston DS, Chenery RJ, Bridges AM: Crystal structure of human cytochrome P450 2D6. J Biol Chem 2006, 281:7614-7622.

16. Porubsky PR, Meneely KM, Scott EE: Structures of human cytochrome P450 2E1: insights into the binding of inhibitors and both small molecular weight and fatty acid substrates. J Biol Chem 2008, 283:33698-33707.

17. Ekroos M, Sjögren T: Structural basis for ligand promiscuity in cytochrome P450 3A4. Proc Natl Acad Sci USA 2006, 103:13682-13687.

18. Yano JK, Wester MR, Schoch GA, Griffin KJ, Stout CD, Johnson EF: The structure of human microsomal cytochrome P450 3A4 determined by Xray crystallography to 2.05-A resolution. J Biol Chem 2004, 279:38091-38094.

19. Szklarz GD, Halpert JR: Molecular basis of P450 inhibition and activation implications for drug development and drug therapy. Drug Metab Dispos 1998, 26:1179-1184.

20. Gotoh O: Cytochrome P450 Family 2 (CYP2) Proteins inferred from Comparative Analyses of Amino Acid and Coding Nucleotide Sequences. J Biol Chem 1992, 267:83-90.

21. Fu DH, Jiang W, Zheng JT, Zhao GY, Li Y, Yi H, Li ZR, Jiang JD, Yang KQ, Wang Y, Si SY: Jadomycin B, an Aurora-B kinase inhibitor discovered through virtual screening. Mol Cancer Ther 2008, 7:2386-2393.

22. Rodrigues AD: Integrated cytochrome P450 reaction phenotyping: attempting to bridge the gap between cDNA-expressed cytochromes P450 and native human liver microsomes. Biochem Pharmacol 1999, 57:465-480.

23. Lu AY, Wang RW, Lin JH: Cytochrome P450 in vitro reaction phenotyping: a re-evaluation of approaches used for P450 isoform identification. Drug Metab Dispos 2003, 31:345-350.

24. Bjornsson TD, Callaghan JT, Einolf HJ, Fischer V, Gan L, Grimm S, Kao J, King SP, Miwa G, Ni L, Kumar G, McLeod J, Obach RS, Roberts S, Roe A, Shah A, Snikeris F, Sullivan JT, Tweedie D, Vega JM, Walsh J, Wrighton SA: The conduct of in vitro and in vivo drug-drug interaction studies a Pharmaceutical Research and Manufacturers of America (PhRMA) perspective. Drug Metab Dispos 2003, 21:815-832.

25. Hickman D, Wang JP, Wang Y, Unadkat JD: Evaluation of the selectivity of In vitro probes and suitability of organic solvents for the measurement of human cytochrome P450 monooxygenase activities. Drug Metab Dispos 1998, 26:207-215.

26. Koley AP, Buters JT, Robinson RC, Markowitz A, Friedman FK: Differential mechanisms of cytochrome P450 inhibition and activation by alphanaphthoflavone. J Biol Chem 1997, 272:3149-3152.

27. McLaughlin LA, Paine MJ, Kemp CA, Maréchal JD, Flanagan JU, Ward CJ, Sutcliffe MJ, Roberts GC, Wolf CR: Why is quinidine an inhibitor of cytochrome P450 2D6? The role of key active-site residues in quinidine binding. J Biol Chem 2005, 280:38617-38624.

28. Guengerich FP, Miller GP, Hanna IH, Sato H, Martin MV: Oxidation of methoxyphenethylamines by cytochrome P450 2D6. Analysis of ratelimiting steps. J Biol Chem 2002, 277:33711-33719.

29. Baune B, Furlan V, Taburet AM, Farinotti R: Effect of Selected Antimalarial Drugs and Inhibitors of Cytochrome P-450 3A4 on Halofantrine Metabolism by Human Liver Microsomes. Drug Metab Dispos 1999, 27:565-568.

30. Murphy PJ: The Development of Drug Metabolism Research as Expressed in the Publications of ASPET: Part 3, 1984-2008. Drug Metab Dispos 2008, 36:1977-1982.
31. Qiu F, Zhu Z, Kang N, Piao S, Qin G, Yao X: Isolation and identification of urinary metabolites of berberine in rats and humans. Drug Metab Dispos 2008, 36:2159-2165.

32. Li YH, Yang P, Kong WJ, Wang YX, Hu CQ, Zuo ZY, Wang YM, Gao H, Gao LM, Feng YC, Du NN, Liu Y, Song DQ, Jiang JD: Berberine analogues as a novel class of the low-density-lipoprotein receptor up-regulators: synthesis, structure-activity relationships, and cholesterol-lowering efficacy. J Med Chem 2009, 52:492-501.

33. Li YH, Li Y, Yang P, Kong WJ, You XF, Ren G, Deng HB, Wang YM, Wang YX, Jiang JD, Song DQ: Design, synthesis, and cholesterol-lowering efficacy for prodrugs of berberrubine. Bioorg Med Chem 2010, 18(17):6422-8.

34. Hua W, Ding L, Chen Y, Gong B, He J, Xu G: Determination of berberine in human plasma by liquid chromatography-electrospray ionization-mass spectrometry. J Pharm Biomed Anal 2007, 44:931-937.

35. Bellosta S, Paoletti R, Corsini A: Safety of Statins: Focus on Clinical Pharmacokinetics and Drug Interactions. Circulation 2004, 109:||I50-III7.

36. Chasman DI, Posada D, Subrahmanyan L, Cook NR, Stanton VP Jr, Ridker PM: Pharmacogenetic study of statin therapy and cholesterol reduction. Jama 2004, 291:2821-2827.

doi:10.1186/1479-5876-9-62

Cite this article as: Li et al:: Bioactivities of berberine metabolites after transformation through CYP450 isoenzymes. Journal of Translational Medicine 2011 9:62.

\section{Submit your next manuscript to BioMed Central and take full advantage of:}

- Convenient online submission

- Thorough peer review

- No space constraints or color figure charges

- Immediate publication on acceptance

- Inclusion in PubMed, CAS, Scopus and Google Scholar

- Research which is freely available for redistribution

Submit your manuscript at www.biomedcentral.com/submit
C Biomed Central 